CLINICAL RESEARCH ARTICLE

\title{
Dried blood spot compared to plasma measurements of blood- based biomarkers of brain injury in neonatal encephalopathy
}

An N. Massaro', Yvonne W. Wu' ${ }^{2}$, Theo K. Bammler ${ }^{3}$, James W. MacDonald ${ }^{3}$, Amit Mathur ${ }^{4}$, Taeun Chang $^{5}$, Dennis Mayock ${ }^{6}$, Sarah B. Mulkey ${ }^{5}$, Krisa van Meurs ${ }^{7}$, Zahra Afsharinejad ${ }^{3}$ and Sandra E. Juul ${ }^{6}$

BACKGROUND: Data correlating dried blood spots (DBS) and plasma concentrations for neonatal biomarkers of brain injury are lacking. We hypothesized that candidate biomarker levels determined from DBS can serve as a reliable surrogate for plasma levels. METHODS: In the context of a phase II multi-center trial evaluating erythropoietin for neuroprotection in neonatal encephalopathy (NE), DBS were collected at enrollment $(<24 \mathrm{~h})$, day 2, 4, and 5. Plasma was collected with the first and last DBS. The relationship between paired DBS-plasma determinations of brain-specific proteins and cytokines was assessed by correlation and Bland-Altman analyses. For analytes with consistent DBS-plasma associations, DBS-derived biomarker levels were related to brain injury by MRI and 1-year outcomes.

RESULTS: We enrolled 50 newborns with NE. While S100B protein, tumor necrosis factor $a$, interleukin (IL) $1 \beta$, IL-6, IL-8 demonstrated significant DBS-plasma correlations, Bland-Altman plots demonstrated that the methods are not interchangeable, with a 2 to 4 -fold error between measurements. No significant relationships were found between DBS levels of TNFa, IL-6, and IL-8 and outcomes.

CONCLUSION: Further work is needed to optimize elution and assay methods before using DBS specimens as a reliable surrogate for plasma levels of candidate brain injury biomarkers in NE.

Pediatric Research (2019) 85:655-661; https://doi.org/10.1038/s41390-019-0298-7

\section{INTRODUCTION}

The search for clinically useful biomarkers of brain injury in neonatal encephalopathy (NE) is ongoing. The hope is that peripheral blood-based biomarkers that reflect end-organ injury will allow for real-time assessment of brain injury progression and treatment efficacy, providing means to individualize neuroprotective care. Several recent investigations have identified candidate brain injury biomarkers in NE. ${ }^{1-5}$ However, large-scale studies are needed to validate the individual and combinative value of candidate biomarkers, since a panel of putative markers will likely be needed for optimal assessment of brain injury risk. To simplify methods for widespread validation, approaches to minimize blood volumes and simplify biorepository methods are needed.

Dried blood spots (DBS) have been used for decades in population-based studies. ${ }^{6-8}$ The reliability of DBS-based analytic methods for certain neonatal conditions is supported by their widespread use in newborn metabolic screening programs, since Guthrie first described methods to identify neonates with phenylketonuria in the 1960 's. ${ }^{8}$ Subsequently, the expansion of newborn metabolic screening via ever advancing analytic methods ${ }^{9}$ has been paralleled by widespread interest in the secondary use of stored DBS specimens for research. ${ }^{10}$ The attraction to a DBS sampling strategy for biomarker studies in neonatal populations is intuitive. The relatively small blood sample volumes required, coupled with the ease and cost-effectiveness of collection, transport, and storage has major advantages when considering studies in newborns. DBS-based methods are particularly attractive for studies of brain injury biomarkers where serial sampling is needed. Given the well-recognized dynamic evolution of neonatal brain injury, ${ }^{11}$ establishing trajectories of candidate biomarkers after insult and neurologic injury is essential. Potential disadvantages include the need for validated analytical methods that can be limited by the variable success of elution and the small volume of biomaterial available for assay. Additionally, given that the standard for clinical chemistry generally relies upon analyses of plasma or serum, whole-blood DBS assays require correlation with plasma/serum levels in order to translate effectively to clinical care.

We previously reported that baseline ( $<24 \mathrm{~h}$ of life) plasma concentrations of $\mathrm{S} 100 \mathrm{~B}$, Tau, Ubiquitin C-terminal hydrolase L1 (UCHL1), interleukin (IL)1 $\beta, \mathrm{IL}-6, \mathrm{IL}-8, \mathrm{IL}-10, \mathrm{IL}-13$, tumor necrosis factor (TNF)a, and interferon (IFN) $Y$ levels were associated with brain injury severity by MRI in newborns with NE. ${ }^{1}$ However, a limitation of establishing the predictive utility of these and other candidate biomarkers is the ability to assess biomarker trajectories over time. In our prior study, most cytokines normalized by day 5 suggesting that the neuroinflammatory response to hypoxiaischemia peaked in the first few days after injury. ${ }^{1}$ For diagnostic and prognostic utility, it is important that reliable biomarkers reflect an individual's evolution of secondary brain injury over

\footnotetext{
${ }^{1}$ Pediatrics - Division of Neonatology, Children's National Health Systems and The George Washington University School of Medicine, Washington, DC, USA; ${ }^{2}$ Neurology and Pediatrics, UCSF, San Francisco, CA, USA; ${ }^{3}$ Department of Environmental \& Occupational Health Sciences, University of Washington, Seattle, WA, USA; ${ }^{4}$ Pediatrics, Washington

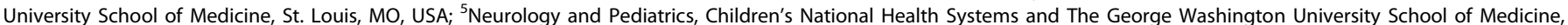
Washington, DC, USA; ${ }^{6}$ Pediatrics-Division of Neonatology, University of Washington, Seattle, WA, USA and ${ }^{7}$ Pediatrics, Stanford, Palo Alto, CA, USA Correspondence: An N. Massaro (anguyenm@cnmc.org)
} 
time. Such investigation would require serial blood sampling, which is resource intensive and raises ethical concerns in high-risk newborns with limited blood volumes. DBS specimens provide a potential answer to these challenges. Several prior studies have used DBS specimens to quantify neonatal cytokine levels and to relate these to outcomes. ${ }^{12-20}$ However, a comparison of plasma with DBS cytokine concentrations in neonates has not been published. Likewise, DBS methods for candidate brain-specific proteins have not been previously reported. The aim of our study was to determine whether candidate biomarker levels determined from DBS specimens can serve as a reliable surrogate for plasma levels. Secondarily, we aimed to assess whether DBS-determined biomarker trajectories relate to outcomes in infants with NE.

\section{METHODS}

Study population

We performed an ancillary study to a phase II multi-center randomized trial evaluating erythropoietin (Epo) for neuroprotection in NE (Neonatal Erythropoietin and Therapeutic Hypothermia Outcomes (NEAT-O) Study; NCT\# 01913340) ${ }^{21}$ to evaluate candidate biomarkers of brain injury. Term or near-term ( $\geq$ 36 weeks gestational age) newborns with moderate or severe encephalopathy and evidence of perinatal depression being treated with hypothermia were prospectively enrolled. The study received institutional review board approval at all hospitals and signed informed consent was obtained from the parent of each participant.

\section{Specimen collection}

Whole-blood specimens $(0.5 \mathrm{~mL})$ were collected in heparinized tubes at baseline (i.e. $<24 \mathrm{~h}$ of life and prior to study drug) and on day of life (DOL) 5 . Specimens were refrigerated at $4{ }^{\circ} \mathrm{C}$ as soon as possible after collection. Specimens were then centrifuged at $6000 \mathrm{rpm}$ for $10 \mathrm{~min}$, with plasma collected and stored at $-80^{\circ} \mathrm{C}$ ideally within $4 \mathrm{~h}$ but allowable up to $48 \mathrm{~h}$ from time of collection. DBS specimens were collected by placing 1 drop of whole blood on filter paper (Whatman 903, GE Healthcare, Westborough, MA) and storing at $-80^{\circ} \mathrm{C}$ with a dessicant after a minimum of $4 \mathrm{~h}$ for complete drying at room temperature. DBS specimens were collected at enrollment ( $<24 \mathrm{~h}$ of life), day 2,3 , and 5 with the first and last sample coinciding with plasma collections.

\section{DBS elution}

For protein elution, the frozen DBS were punched using $10-\mathrm{mm}$ disposable biopsy AcuPunch (Acuderm, Inc., Fort Lauderdale, FL). The punched paper specimen was submerged in $400 \mu \mathrm{L}$ of PBSbased buffer containing 0.1\% Triton (Sigma Chemical Co.Aldrich, St. Louis, MO) and $0.03 \%$ Tween-20 (Fisher, Hampton, NH) vortexed for $30 \mathrm{~s}$ and incubated on a shaker for $1 \mathrm{~h}$ at $4{ }^{\circ} \mathrm{C}$. The eluted blood samples were aliquoted and stored frozen at $-80^{\circ} \mathrm{C}$ until analyzed. All measurements were adjusted for total protein extracted from each spot. The Pierce BCA Protein Assay Kit (Thermo Fisher Scientific, Waltham, MA) was used to determine protein concentrations according to the manufacturer's recommended protocol.

Biomarker determinations

Brain-specific proteins Tau, UCHL1, and neuron-specific enolase (NSE) levels were quantified via a commercially available assay kit (Human Neurological Disorders Magnetic Bead Panel 1, EMD Millipore; Germany) on the multiplexed bead-based ELISA Luminex platform (Luminex Corp., Austin, TX). Glial fibrillary acidic protein (GFAP), brain derived neurotrophic factor (BDNF), and Monocyte Chemoattractant Protein-1 (MCP-1) were measured using a custom multiplexed electrochemiluminescence-based assay (Meso Scale Discovery; Rockville, MD). ${ }^{22}$ S100B protein was assayed separately using a commercially available ELISA (Human S100B ELISA kit, Millipore; Germany). Inflammatory cytokines IFN $\gamma$, TNFa, IL-1 $\beta$, IL-6, IL-8, IL-10, IL-12p70, and IL-13 and Epo levels were determined using the Meso Scale Discovery platform (V-PLEX Proinflammatory Panel 1 and Human EPO Base Kit respectively, Meso Scale Discovery, Rockville, MD). Details of assay characteristics are summarized in Table 1. All assays were performed in duplicate and results averaged for analyses.

Neurological outcomes

Magnetic resonance imaging (MRI) and functional outcomes of the study cohort have been reported previously. ${ }^{21}$ Brain injury severity was classified as none, mild, moderate, or severe by brain

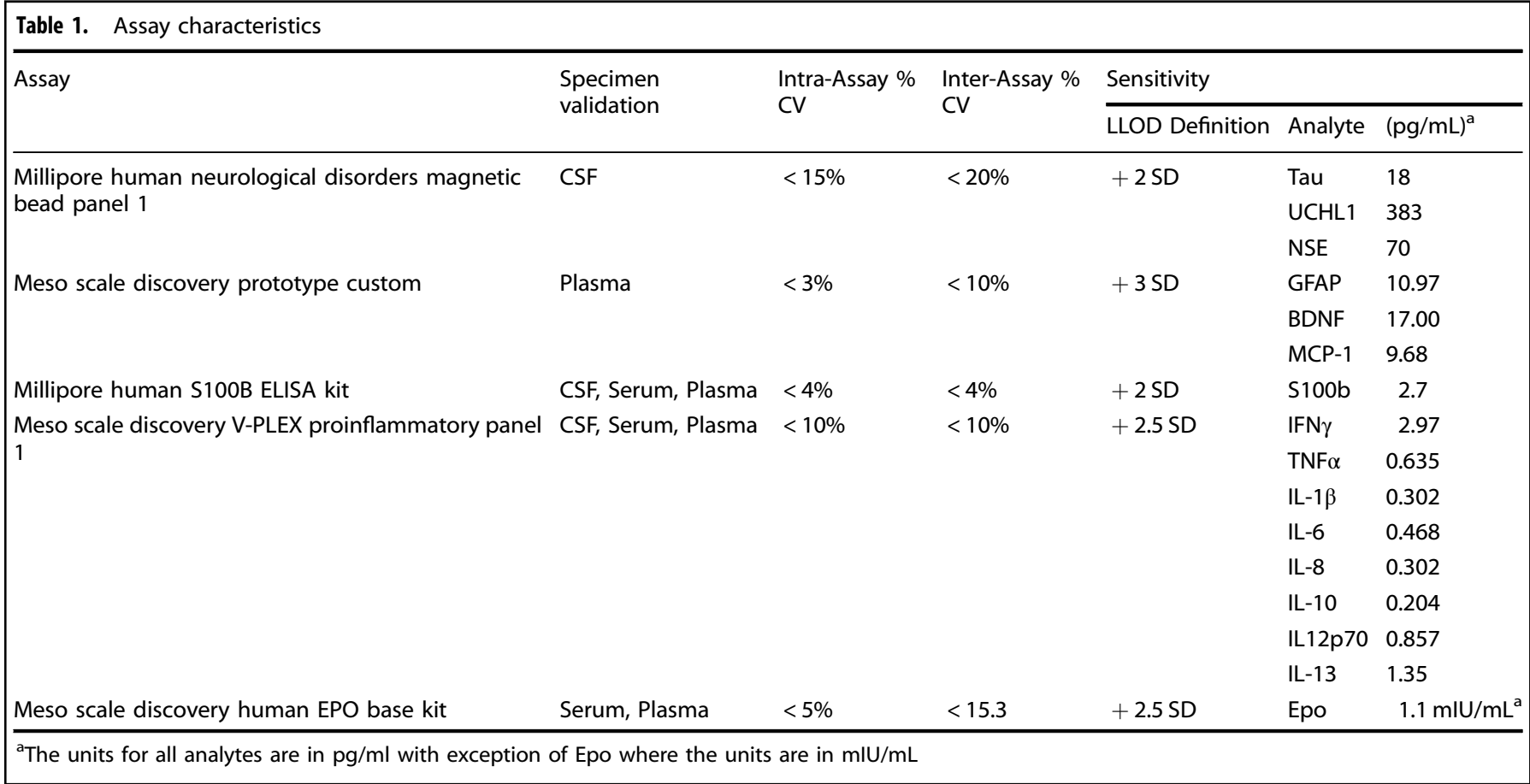


MRI performed as part of routine clinical care at 4-7 days of age. ${ }^{23}$ Early neurodevelopmental outcomes were assessed at 1 year with the Alberta Infant Motor Scale (AIMS) ${ }^{24,25}$ and the Warner Initial Developmental Evaluation (WIDEA). ${ }^{26,27}$ Adverse neurological outcome was defined as (a) death or severe brain injury by MRI and (b) death or moderate to severe neurodevelopmental impairment (i.e., AIMS less than 5th percentile for age, and WIDEA more than 2 standard deviations below the normative mean).

Statistical analysis

Exploratory data analyses included quantification of samples below detection limits and exclusion of analytes with high (>60\%) missing data. After log transformation, linear regression analyses were performed to assess the association of simultaneously collected DBS and plasma samples for each analyte. For analytes with high DBS-plasma correlations, Bland-Altman (BA) plots were performed to compare DBS-based predicted plasma levels and directly measured plasma levels. The BA analysis quantifies agreement between two measurements by constructing limits of agreement (LOA) defined by the mean and the standard deviation $(s)$ of the differences between two measurements. $^{28}$ The LOA is defined as a $95 \%$ confidence interval for the difference between the two methods. Clinically acceptable agreement is defined by the width or range of the LOA to represent a biologically meaningful difference. We considered a maximum of 2-fold difference as clinically acceptable based on prior work evaluating DBS-plasma agreement. ${ }^{29}$ Intrasubject correlations were not considered in these analyses. Biomarkers with significant DBS-plasma correlations were related to neurological outcomes using logistic regression analyses, testing first for any time-dependent associations, with a random intercept for each subject, followed by comparisons at each time point. Sample size of 50 subjects was determined by the primary trial and sampling for plasma and DBS specimens was based on pragmatic (i.e., to coincide with clinical laboratory monitoring) and ethical (i.e., limited sampling frequency acceptable for critically ill newborns) considerations. Based on entering into analyses with an expected 100 paired DBS-plasma observations, post-hoc power assessment was consistent with adequate sample size to detect a $95 \% \mathrm{Cl}$ of $\pm 0.34 \mathrm{s.}^{30}$

\section{RESULTS}

Fifty term or near-term neonates with moderate to severe encephalopathy were enrolled at 7 centers between December 2013 and November 2014. Detailed characteristics of the study population stratified by treatment group, outcomes, as well as plasma biomarker data have been previously reported. ${ }^{1,21}$ Briefly, mean \pm SD birthweight was $3.3 \pm 0.7 \mathrm{~kg}$, gestational age $38.7 \pm$ 1.7 weeks, $52 \%$ were male, and $18 \%$ presented with severe encephalopathy. Death occurred in 7 (14\%) patients. All surviving infants had an MRI and severe brain injury by MRI was observed in $6(12 \%)$. Of the 43 surviving infants, 41 infants were evaluated at 1 year and $7(17 \%)$ infants were classified as having moderate to severe neurodevelopmental impairment evidenced by WIDEA or AIMs evaluation at a median age of $12.7 \pm 0.7$ months. Baseline plasma and DBS samples were drawn from all patients at median age of $16.2 \mathrm{~h}$ of age (range 3.3-23.9 h). One infant died on day of life (DOL) 2 and 3 infants died prior to DOL 5. DBS specimens were drawn from 49 patients on DOL 2 at median age of $42 \mathrm{~h}$ (range 27.3-48.9 h) and 48 patients on DOL 3 at median age of $64.8 \mathrm{~h}$ (range $52-74.6 \mathrm{~h}$. DOL 5 plasma and DBS samples were obtained in the 47 surviving infants at median age of $112.8 \mathrm{~h}$ (range 96-120 h).

Several analytes (Tau, UCHL1, NSE, BDNF, GFAP, MCP-1, IFNy, IL10, IL-12p70, and IL-13) were excluded from further analysis due to DBS levels below the limits of detection in $>60 \%$ of samples. Of the remaining analytes, TNFa, IL- $6, \mathrm{IL}-8$ and Epo had the most consistent relationship between DBS and plasma levels (Fig. 1). While DBS and fresh plasma measurements for these analytes are highly correlated, Bland-Altman plots indicate that DBS and plasma measurements are not interchangeable (Fig. 2). In all cases, the 2 SD range is larger than a log-fold change of 1 , indicating a two to four fold difference between DBS and plasma measurements. TNFa had the least variation between DBS and plasma levels, while IL $1 \mathrm{~b}$ and S100B had the greatest differences.

Trajectories of TNFa, IL-6, and IL-8 were further explored in relation to neurological outcomes. All three cytokines decreased over time. No significant relationships were found between DBS levels of TNFa, IL-6, and IL-8 with death or severe brain injury by MRI ( $p>0.05$, Fig. 3a). Similarly, cytokine trajectories by DBS were not predictive of death or neurodevelopmental impairment at 1 year of age ( $p>0.05$, Fig. $3 b)$.

\section{DISCUSSION}

While DBS-plasma correlations were high, we demonstrate a 2-fold or greater difference between DBS and plasma-based determinations of candidate brain injury biomarkers. That this variability represents a biologically meaningful difference is supported by the fact that no relationship was observed between DBS levels of IL-6, IL-8, and TNFa and neurological outcomes in babies with NE. This may be in contrast to our prior report of correlation between plasma levels of IL-6, IL-8, and TNF a and MRI outcomes in this cohort. It should be noted that we did not observe correlations between either plasma or DBS measurements of these analytes and one-year neurodevelopmental outcomes. ${ }^{1}$ These findings raise caution around using DBS methods for larger validation studies of candidate biomarkers of brain injury in NE until elution and assay methods are optimized. While a DBS-based sampling strategy may offer an attractive means for repeated measurements in a single subject, our data suggest that this approach is not reliable using available multiplexed assays for brain-specific proteins and cytokines of interest investigated in this study.

While interest in clinical and research use of DBS has expanded over the past several decades, ${ }^{9,10}$ it should be recognized that the list of validated DBS assays that have demonstrated acceptable levels of agreement with serum or plasma-based results is limited. In addition to thyroxine, thyrotropin, and other common assays included in newborn metabolic screening, other analytes that have been extensively studied and correlated with venous blood samples include c-reactive protein, sex and steroid hormones, and HIV and hepatitis antibodies. ${ }^{7}$ One study evaluated IL- 6 and reported acceptable agreement between DBS and plasma levels with Bland-Altman 95\% LOA of -1.23 to 1.04 . However, it should be noted that this study used samples from 46 adult volunteers and all measured levels of IL- 6 were $<7 \mathrm{pg} / \mathrm{mL}$. ${ }^{29}$ To our knowledge, no studies have reported plasma/DBS validation of other cytokines or brain-specific proteins in babies with NE.

DBS assay validation against plasma levels is important as several factors can influence measurement of a particular analyte from DBS.7 As DBS samples are constituted from whole blood, it is important to determine whether a particular assay is influenced by the presence of cellular components. Interference preventing quantification is rare ${ }^{31}$ and not previously reported for neonatal cytokines so we did not adjust for this factor in our analyses. Given relatively low sample volumes provided from a DBS (a $3.2 \mathrm{~mm}$ DBS punch will yield $\sim 1.4 \mu \mathrm{l}$ of blood), ${ }^{32}$ assays developed for serum/plasma may not have adequate sensitivity to analyze DBS samples, particularly if high volumes of undiluted serum/plasma are required. In particular, it should be noted that the assay utilized for UCHL1, NSE, and Tau analyses was validated for cerebral spinal fluid (Table 1) and, therefore, the inability to quantify levels from DBS is not surprising. Finally, the analyte must be reliably and consistently eluted from the filter 


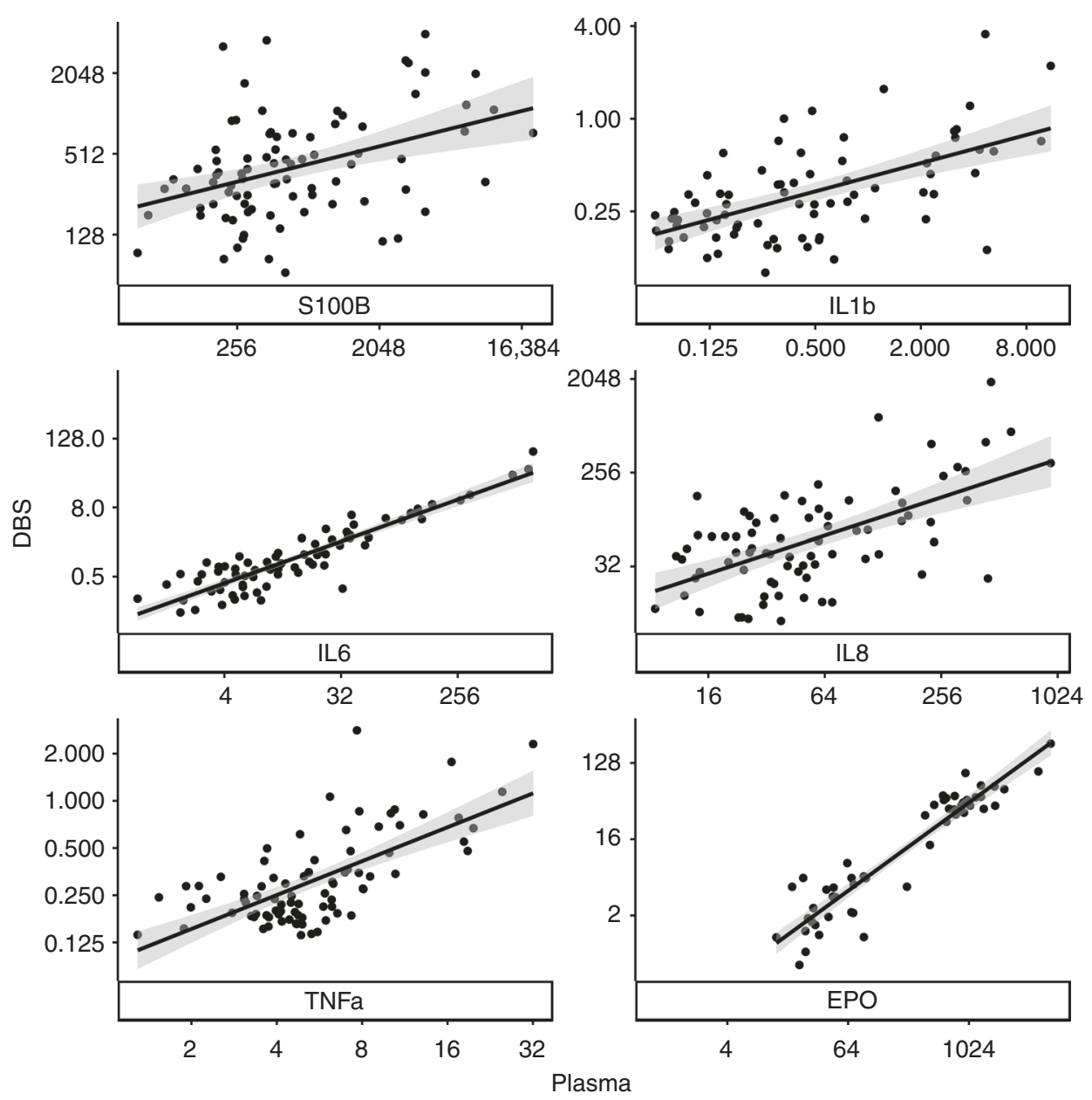

Fig. 1 Association between DBS and plasma concentrations of candidate biomarkers by linear regression (solid line). Ninety-seven paired observations are included ( $n=50$ at baseline and $n=47$ on day 5). The dark gray bands around the regression lines represent the standard error for the regression line. Data presented after logarithmic transformation

paper. Elution protocols can vary in the components of buffer solution used, duration of incubation, shaking/agitation strategy, and temperature. While we performed some preliminary experiments evaluating alternative incubation, shaking, and concentration methods to optimize the elution protocol (data not shown), we ultimately relied upon previously described elution methods for neonatal cytokines. ${ }^{15}$ Future studies are needed to evaluate whether optimized elution and assay protocols will improve the reliability of DBS-based methods for brain-specific protein recovery. This is particularly important as several of the analytes evaluated in the current study, including Tau, UCHL1, NSE, GFAP, IFNy, IL-10, IL-12p70, and IL-13, were unable to be reliably quantified from DBS.

Despite the lack of studies establishing agreement between DBS and plasma-based neonatal cytokine measurements, several studies have evaluated DBS-derived neonatal cytokine levels and neurological outcomes. Nelson et al reported that neonatal levels of IL-1 $\beta$, IL-8, IL-9, TNFa, and RANTES were elevated in mostly term-born children with cerebral palsy compared to controls. ${ }^{20}$ Bartha et al. described a relationship between elevated IL-1 $\beta, I L-6, I L-8, I L-9$, and TNFa in encephalopathic neonates and abnormal neurodevelopmental outcomes at 30 months of age. ${ }^{13}$ Additionally, recent studies have demonstrated a relationship between IL-6 polymorphisms and cerebral palsy. ${ }^{33,34}$ While these authors reported a relationship between neonatal cytokines and presence of lactate in the deep gray nuclei by magnetic resonance spectroscopy, they did not observe a relationship with qualitative scoring of MRI injury, similar to our findings. These two studies used recycling immunoaffinity chromatography for cytokine measurements. Although different from the electochemiluminescence-based immunoassays used in our study, both approaches have comparable sensitivity and dynamic range. Investigators of the Extremely Low Gestational Age Newborn (ELGAN) Study have also used electrochemiluminescence-based immunoassays to measure neonatal cytokines from DBS and reported relation to outcomes in preterm infants. ${ }^{16-18}$ More recently, a population-based cohort study reported no relationship between DBS-based neonatal cytokine measures and developmental delay at 36 months. ${ }^{35}$ It is possible that our results corroborate the notion that neonatal cytokines lack specificity for predicting brain injury in newborns. However, it is unclear from our study whether these variable findings are due to lack of association between neonatal cytokine levels and outcomes, or the potential for measurement error introduced by the DBS method.

The use of newer or alternative technologies such as mass spectrometry coupled to high performance liquid or gas chromatography and capillary electrophoresis have also been used to provide non-invasive determinations using very small volumes of biofluids (e.g., urine, saliva, amniotic fluid). Recent papers have validated these techniques for targeting oxidative damage byproducts from fatty acid components of the brain (i.e., docosahexaenoic acid, adrenic acid). ${ }^{36-39}$ Whether employing such alternative analytical methodologies may yield improved performance of DBS-determined biomarker levels is an area for future study. 

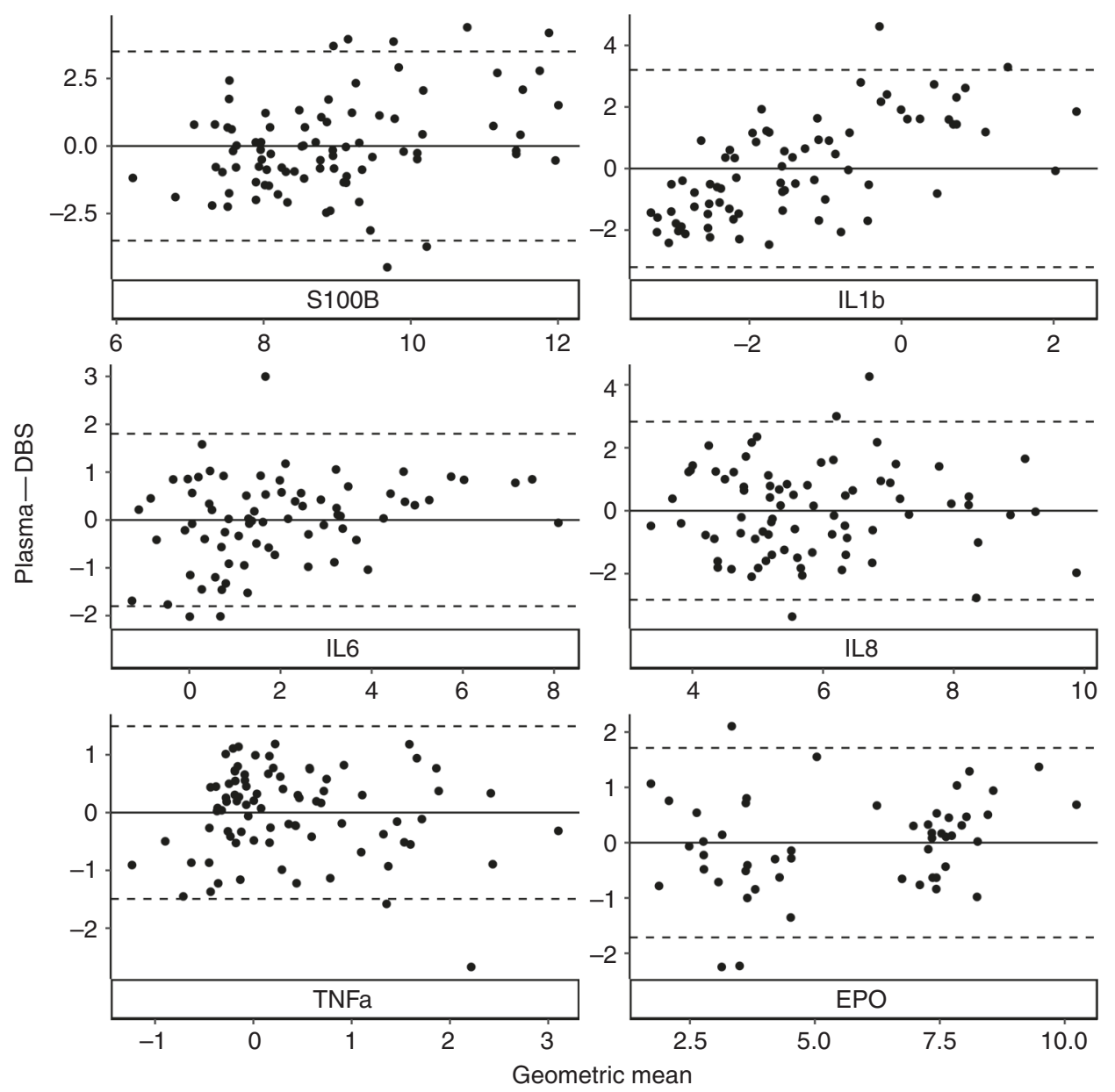

Fig. 2 Bland-Altman plots of DBS-predicted plasma levels versus observed plasma levels. $y$-axis represents the log-fold change (i.e., log ratio) between DBS and plasma levels. Ninety-seven paired observations are included ( $n=50$ at baseline and $n=47$ on day 5). The solid horizontal line in the Bland-Altman plots indicates the mean log difference between DBS and plasma, and the dashed horizontal lines indicate \pm 2 standard deviations (SD)
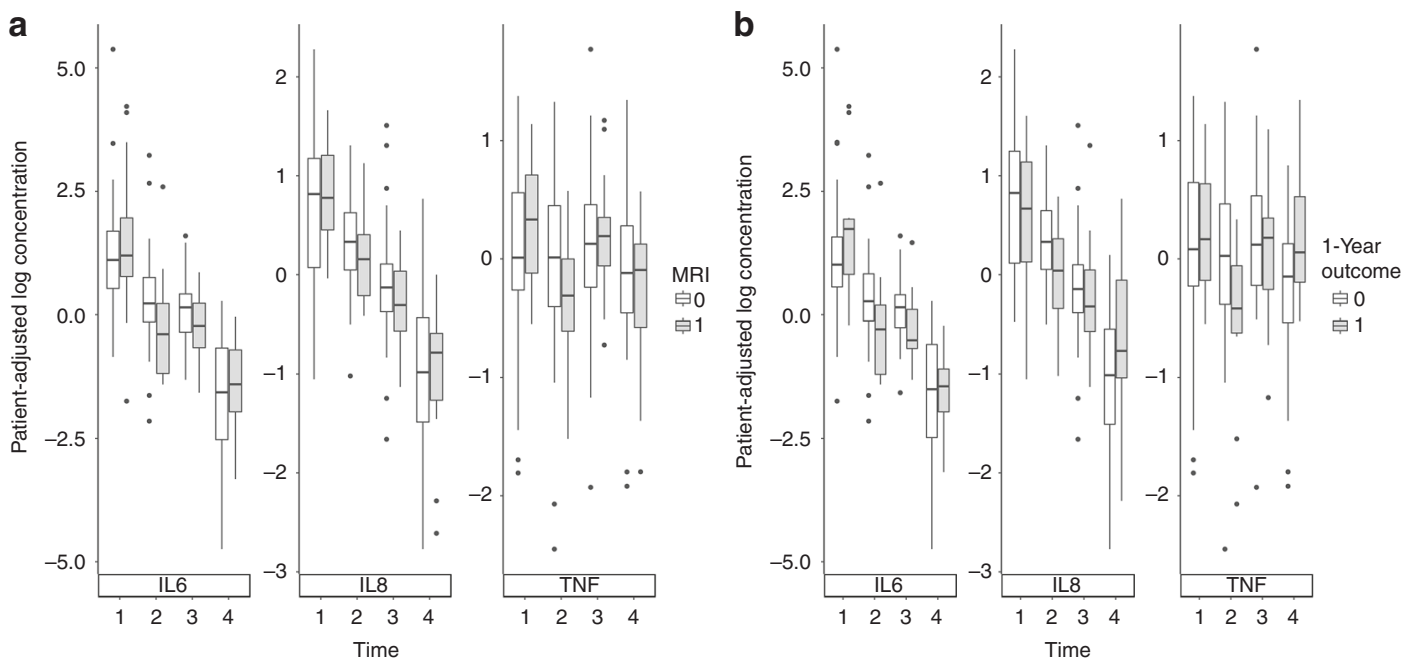

Fig. 3 Log-transformed concentrations of IL-6, IL-8, and TNF $\alpha$ over time. Data are adjusted for patient-specific levels (i.e., intrasubject correlations) and stratified by outcomes defined by a death or severe brain injury by MRI $(n=50)$ and $\mathbf{b}$ functional outcomes at 1 year ( $n=47$ ). Box plots represent median and interquartile ranges of cytokine levels from infants with favorable (white; "0") compared to adverse (gray; " 1 ") outcomes 
Our study has several limitations. Sample size for this ancillary study was determined by the parent randomized trial and therefore limited our ability to adjust for additional potential confounders or for multiple comparisons. We did not adjust for repeated measures in our BA analyses. Repeated sampling within subject was explored by Bland and Altman, in which they showed, as expected, that repeated measures would tend to reduce the estimated variance, thereby reducing the LOA. ${ }^{40}$ Thus, our LOA ranges may be underestimated, further supporting the concern regarding agreement between the two methods. While all sites were provided study-specific standard operating procedures for specimen collection and handling, it is possible that variability in specimen processing and storage may have affected results. For example, collection of blood by heelstick (particularly on day 5 when indwelling lines were often removed) may have introduced variability given the varying amounts of interstitial fluid that can be obtained with capillary blood. We limited the number of freeze thaw cycles as practicably possible. All samples were thawed once to aliquot for individual planned assays, refrozen, and then ultimately thawed for analysis. Given all samples underwent the same number of freeze/thaw cycles, this was unlikely to introduce variability in our analyses and prior work has established stability of cytokines after freezing. ${ }^{41}$ Additionally, further work to optimize elution protocols may yield different results as mentioned previously. Finally, we assessed brain injury by MRI and developmental outcomes at 1 year. While neonatal MRI is a reliable predictor of neurodevelopmental outcomes in newborns with $\mathrm{NE}^{42,43}$ it is possible that our findings may differ if longer term functional outcomes were assessed. Given these limitations, it is recognized that DBS methods may still have utility in larger studies if assays can be adequately optimized and preliminary studies can demonstrate reliability either through acceptable relationship to plasma measurements and/or to outcomes of interest. However, our study raises caution regarding the use of current DBS methods for large-scale investigation of brain injury biomarkers in babies with $\mathrm{NE}$, particularly when using multiplexed assays developed and optimized for plasma samples.

\section{ACKNOWLEDGEMENTS}

We thank Jessica Kan Vedder for her devoted organizational support of the study, and Ron McPherson for his assistance with biorepository maintenance. We thank Michael Msall for his expertize, guidance and training in the use of the AIMS and WIDEA neurodevelopmental evaluations. The authors greatly appreciate the work of the Data Safety Monitoring Board: Robin Ohls (Chair), Janet Soul and John Barks.

\section{FUNDING}

This study was funded by the Cerebral Palsy Alliance Research Foundation and the Thrasher Research Fund.

\section{AUTHOR CONTRIBUTIONS}

A.N.M. made substantial contributions to conception and design of the study and interpretation of data; prepared the first draft of the article and revised it critically for important intellectual content; and approved of the version to be published. Y.W.W., T.K.B., and S.J. made substantial contributions to conception and design of the study, and interpretation of data; revised the article critically for important intellectual content; and approved of the version to be published. J.W.M made substantial contributions to the analysis and interpretation of data; revised the article critically for important intellectual content; and approved of the version to be published. A.M., T.C., D.M., S.B.M., K.v.M., and Z.A. made substantial contributions to the acquisition of data, revised the article critically for important intellectual content; and approved of the version to be published.

\section{ADDITIONAL INFORMATION}

Competing interests: The authors declare no competing interests.

Publisher's note: Springer Nature remains neutral with regard to jurisdictional claims in published maps and institutional affiliations.

\section{REFERENCES}

1. Massaro, A. N. et al. Plasma biomarkers of brain injury in neonatal hypoxicischemic encephalopathy. J. Pediatr. 194, 67-75 (2018). e1.

2. Massaro, A. N. et al. Biomarkers of MRI brain injury in neonatal hypoxic ischemic encephalopathy treated with whole body hypothermia-a pilot study. Pediatr. Crit. Care Med. 14, 310-317 (2013).

3. Massaro, A. N. et al. Biomarkers of brain injury in neonatal encephalopathy treated with hypothermia. J. Pediatr. 161, 434-440 (2012).

4. Chalak, L. F. et al. Biomarkers for severity of neonatal hypoxic-ischemic encephalopathy and outcomes in newborns receiving hypothermia therapy. J. Pediatr. 164, 468-474 (2014).

5. Orrock, J. E. et al. Association of brain injury and neonatal cytokine response during therapeutic hypothermia in newborns with hypoxic-ischemic encephalopathy. Pediatr. Res. 79, 742-747 (2016).

6. McDade, T. W. Development and validation of assay protocols for use with dried blood spot samples. Am. J. Human. Biol. 26, 1-9 (2014).

7. McDade, T. W., Williams, S. \& Snodgrass, J. J. What a drop can do: dried blood spots as a minimally invasive method for integrating biomarkers into populationbased research. Demography 44, 899-925 (2007).

8. Guthrie, R. \& Susi, A. A simple phenylalanine method for detecting phenylketonuria in large populations of newborn infants. Pediatrics 32, 338-343 (1963).

9. Bailey, D. B. Jr. \& Gehtland, L. Newborn screening: evolving challenges in an era of rapid discovery. JAMA 313, 1511-1512 (2015).

10. Therrell, B. L. Jr et al. Committee report: considerations and recommendations for national guidance regarding the retention and use of residual dried blood spot specimens after newborn screening. Genet. Med. 13, 621-624 (2011).

11. Ferriero, D. M. Neonatal brain injury. N. Engl. J. Med. 351, 1985-1995 (2004).

12. Hecht, J. L. et al. Relationship between neonatal blood protein concentrations and placenta histologic characteristics in extremely low GA newborns. Pediatr. Res. 69, 68-73 (2011).

13. Bartha, A. I. et al. Neonatal encephalopathy: association of cytokines with MR spectroscopy and outcome. Pediatr. Res. 56, 960-966 (2004).

14. Skogstrand, K. et al. Simultaneous measurement of 25 inflammatory markers and neurotrophins in neonatal dried blood spots by immunoassay with xMAP technology. Clin. Chem. 51, 1854-1866 (2005).

15. Leviton, A. et al. Early postnatal blood concentrations of inflammation-related proteins and microcephaly two years later in infants born before the 28th postmenstrual week. Early Hum. Dev. 87, 325-330 (2011).

16. O'Shea, T. M. et al. Inflammation-initiating illnesses, inflammation-related proteins, and cognitive impairment in extremely preterm infants. Brain Behav. Immun. 29, 104-112 (2013).

17. O'Shea, T. M. et al. Elevated blood levels of inflammation-related proteins are associated with an attention problem at age 24 mo in extremely preterm infants. Pediatr. Res. 75, 781-787 (2014).

18. O'Shea, T. M. et al. Elevated concentrations of inflammation-related proteins in postnatal blood predict severe developmental delay at 2 years of age in extremely preterm infants. J. Pediatr. 160, 395-401 (2012).

19. Nelson, K. B. et al. Neuropeptides and neurotrophins in neonatal blood of children with autism or mental retardation. Ann. Neurol. 49, 597-606 (2001).

20. Nelson, K. B., Dambrosia, J. M., Grether, J. K. \& Phillips, T. M. Neonatal cytokines and coagulation factors in children with cerebral palsy. Ann. Neurol. 44, 665-675 (1998).

21. Wu Y. W., et al. High-Dose Erythropoietin and Hypothermia for Hypoxic-Ischemic Encephalopathy: A Phase II Trial. Pediatrics 137, e2 0160191 (2016).

22. Buonora, J. E. et al. Multivariate analysis of traumatic brain injury: development of an assessment score. Front. Neurol. 6, 68 (2015).

23. Bednarek, N. et al. Impact of therapeutic hypothermia on MRI diffusion changes in neonatal encephalopathy. Neurology 78, 1420-1427 (2012).

24. Piper, M. C., Pinnell, L. E., Darrah, J., Maguire, T. \& Byrne, P. J. Construction and validation of the Alberta Infant Motor Scale (AIMS). Can. J. Public Health 83(Suppl 2), S46-S50 (1992)

25. Darrah, J., Piper, M. \& Watt, M. J. Assessment of gross motor skills of at-risk infants: predictive validity of the Alberta Infant Motor Scale. Dev. Med. Child Neurol. 40, 485-491 (1998).

26. Msall, M. T. M. \& Ottenbacher, K. J. Functional assessment of preschool children: optimizing developmental and family supports in early intervention. Infants Young-. Child. 14, 16-66 (2001).

27. Msall, M. E. Measuring functional skills in preschool children at risk for neurodevelopmental disabilities. Ment. Retard. Dev. Disabil. Res. Rev. 11, 263-273 (2005).

28. Bland, J. M. \& Altman, D. G. Statistical methods for assessing agreement between two methods of clinical measurement. Lancet 1, 307-310 (1986).

29. Miller, E. M. \& McDade, T. W. A highly sensitive immunoassay for interleukin-6 in dried blood spots. Am. J. Human. Biol. 24, 863-865 (2012).

30. Phatak, A. G. \& Nimbalkar, S. M. Method comparison (agreement) studies: myths and rationale. J. Clin. Diagn. Res. 11, JI01-JI03 (2017). 
31. Ahluwalia, N., Lonnerdal, B., Lorenz, S. G. \& Allen, L. H. Spot ferritin assay for serum samples dried on filter paper. Am. J. Clin. Nutr. 67, 88-92 (1998).

32. Mei, J. V., Alexander, J. R., Adam, B. W. \& Hannon, W. H. Use of filter paper for the collection and analysis of human whole blood specimens. J. Nutr. 131, 1631S-1636SS (2001).

33. Khankhanian, P. et al. Sequencing of the IL6 gene in a case-control study of cerebral palsy in children. BMC Med. Genet. 14, 126 (2013).

34. Wu, Y. W. et al. Interleukin- 6 genotype and risk for cerebral palsy in term and near-term infants. Ann. Neurol. 66, 663-670 (2009).

35. Ghassabian, A. et al. Concentrations of immune marker in newborn dried blood spots and early childhood development: results from the Upstate KIDS Study. Paediatr. Perinat. Epidemiol. 32, 337-345 (2018).

36. Kuligowski, J. et al. Analysis of lipid peroxidation biomarkers in extremely low gestational age neonate urines by UPLC-MS/MS. Anal. Bioanal. Chem. 406, 4345-4356 (2014).

37. Kuligowski, J. et al. Urinary lipid peroxidation byproducts: are they relevant for predicting neonatal morbidity in preterm infants? Antioxid. Redox Signal. 23, 178-184 (2015).
38. Chafer-Pericas, C. et al. Mass spectrometric detection of biomarkers for early assessment of intraamniotic fluid infection. Data Brief. 5, 1026-1030 (2015).

39. Rausell D., et al Newly validated biomarkers of brain damage may shed light into the role of oxidative stress in the pathophysiology of neurocognitive impairment in dietary restricted phenylketonuria patients. Pediatr. Res. 2018. https://doi.org/ 10.1038/s41390-018-0202-x. (epub ahead of print)

40. Bland, J. M. \& Altman, D. G. Agreement between methods of measurement with multiple observations per individual. J. Biopharm. Stat. 17, 571-582 (2007).

41. Keustermans, G. C., Hoeks, S. B., Meerding, J. M., Prakken, B. J. \& de Jager, W. Cytokine assays: an assessment of the preparation and treatment of blood and tissue samples. Methods 61, 10-17 (2013).

42. Shankaran, S. et al. Brain injury following trial of hypothermia for neonatal hypoxic-ischaemic encephalopathy.Arch. Dis. Child Fetal Neonatal Ed. 97, F398-F404 (2012).

43. Rutherford, M. et al. Assessment of brain tissue injury after moderate hypothermia in neonates with hypoxic-ischaemic encephalopathy: a nested substudy of a randomised controlled trial. Lancet Neurol. 9, 39-45 (2010). 